\section{Chandrasekhar on Eddington}

Fred Hoyle

Eddington: The Most Distinguished

Astrophysicist of his Time.

By S. Chandrasekhar

Cambridge University Press: 1983. Pp.64. £7.50, \$12.50.

ARTHUR Stanley Eddington was born on 28 December, 1882. Two centenary lectures were given in his memory, and the Master and Council of Trinity College, Cambridge, made the happy decision of asking Professor Chandrasekhar to deliver them. To this assignment he brought to bear all the care and scholarship which has attended his work down the years. The first of his lectures bears the same name as the book itself; the second is entitled "Eddington: The Expositor and the Exponent of General Relativity'.

The first lecture has five sections: parts I and II are biographical, III is concerned with Eddington's early researches on stellar motions, IV is on stellar structure, and $\mathrm{V}$ is an amusing summary of Eddington's relations with his contemporaries, relations both friendly and stormy. The work on stellar structure, the internal constitution of the stars as Eddington preferred to call it, was of such massive proportions that I must pass it by except for the following brief sketch. Of the factors on which the luminous output of a star depends, Eddington showed that by far the most important is its mass. Given the mass it was possible to predict, without any knowledge of the source of a star's energy, what the luminous output must be, and to do so with a degree of accuracy that was considered remarkable in the 1920 s.

Even within the short space available in a lecture, Professor Chandrasekhar brings out the remarkable degree to which Eddington was self-contained in his work, less influenced by others immediately around him than is usual for a scientist and reminding us of Peer Gynt's motto: To thyself be sufficient.

The second lecture has nine sections. Part I is a brief description of the origins of Einstein's theory, II is concerned with Eddington's early enthusiasm for the theory and his part in organizing the eclipse expeditions to test the predicted bending of light past the Sun, and III contains Professor Chandrasekhar's own recollections about this episode in Eddington's life. I was amused to find this problem: "A affirms that $B$ denies that $C$ declares that $D$ is a liar" rearing its head again, a problem which last tormented me in the year 1936 when I attended Eddington's lecture course, "The Combination of Observations". There were turbulent arguments among us students about it, and frankly speaking I never could see that a

for many years, it is hardly a satisfactory answer that the judgement lies in the reactions of a man's contemporaries. The issue is better related to fact. Where facts are many or are strong, it is possible to be sure. Where they are few, and are subject to a variety of interpretations, sureness becomes cocksureness, which unfortunately one must agree was the situation in Eddington's later work.

Nevertheless, one word of defence! Is Eddington's cosmology really negated because of the discovery of primordial helium and the microwave background? With a non-zero cosmical constant, one can have an initial big-bang that is adjusted by a special choice of parameters to pass asymptotically close to the Einstein static state, and with this special choice Eddington could have defended himself, as indeed Lemaitre did. Discovery of the existence of primordial helium and the microwave background date from 1965. Is it not a little ominous for those who are so cocksure about their ideas in cosmology today that the factual evidence for their position has remained nearly static over almost two decades?

Sir Fred Hoyle was an undergraduate student of Arthur Eddington's at the University of Cambridge in 1935-1936.

\section{Son of John Paul}

\section{G.D. Clarke}

\section{Culture of Animal Cells: A Manual of}

Basic Technique.

By R. Ian Freshney.

Alan R. Liss/Wiley: 1983. Pp.295.

$\$ 49.50, £ 33$.

THE washing of glassware, media making and cell production may seem generations away from gene cloning, monoclonal antibodies and fluorescent-activated cell sorting. They are not of course; but in large laboratories they are a central service and, if efficiently carried out, those who perform them may not be readily identified in corridors or canteens. In a decade of rapid advances, especially in molecular genetics, such services may be undervalued, but never by one used to a lessfavoured environment.

The major part of Ian Freshney's book covers the setting up of a cell culture facility and the routine methods necessary to its function. The author then gives brief protocols for the more specialized techniques, referring always to the original publications. The bibliography is excellent and the cross-referenced list of equipment and reagents with addresses of manufacturers (in Europe and North America) will prove useful to many. The employment of trained staff, or advice from experienced colleagues, is preferable to book learning for those setting up a culture unit from scratch; but this book, with its large format, Scientific American lucidity, and generous use of diagrams, will be an essential adjunct.

The protocols, which have been taken direct from the Methods Book of a versatile laboratory, must be almost foolproof. Nevertheless, novices looking only for one particular procedure would be advised to spend some hours on a general browse. History records that many wrinkles and pitfalls are only obvious after expensive and time-consuming experience; Ian Freshney mentions them all somewhere but they could be constantly repeated.

Common sense suggests that the sections on organization and routine procedures will be of greatest use in developing countries, where budgets are tight and labour cheap. So a short appendix on alternative technology might have been included, while the absence of reference to probabilistic events in the sections on cell synchrony and physical cell separation could lead biochemists into an inappropriate use of cell populations. But these are small factors in a book invaluable in a wide range of rapidly expanding fields. The simple presentation of established routines, and introduction to the invaluable serum-free media of Ham and Sato, and to modern molecular techniques, make this a worthy competitor for Professor Paul's classic of 1959-1975.

G.D. Clarke is in the Cell Proliferation Studies Laboratory of the Imperial Cancer Research Fund, Lincoln's Inn Fields, London. 